\title{
Residual Recurrent CRNN for End-to-End Optical Music Recognition on Monophonic Scores
}

\author{
Aozhi Liu \\ liuaozhi201310@163.com \\ Ping An Technology(Shenzhen) \\ Co.,Ltd \\ China \\ Baoqiang Han \\ hanbaoqiang038@pingan.com.cn \\ Ping An Technology(Shenzhen) \\ Co.,Ltd \\ China
}

\author{
Lipei Zhang* \\ oscarzlp@outlook.com \\ University College London \\ United Kingdom
}

\author{
Zifeng Cai \\ caizifeng709@pingan.com.cn \\ Ping An Technology(Shenzhen) \\ Co.,Ltd \\ China \\ Jing Xiao \\ xiaojing661@pingan.com.cn \\ Ping An Technology(Shenzhen) \\ Co.,Ltd \\ China
}

\author{
Yaqi Mei \\ meiyaqi381@pingan.com.cn \\ Ping An Technology(Shenzhen) \\ Co.,Ltd \\ China
}

Zhaohua Zhu

zhuzhaohua582@pingan.com.cn

Ping An Technology(Shenzhen)

Co.,Ltd

China

\begin{abstract}
One of the challenges of the Optical Music Recognition task is to transcript the symbols of the camera-captured images into digital music notations. Previous end-to-end model which was developed as a Convolutional Recurrent Neural Network does not explore sufficient contextual information from full scales and there is still a large room for improvement. We propose an innovative framework that combines a block of Residual Recurrent Convolutional Neural Network with a recurrent Encoder-Decoder network to map a sequence of monophonic music symbols corresponding to the notations present in the image. The Residual Recurrent Convolutional block can improve the ability of the model to enrich the context information. The experiment results are benchmarked against a publicly available dataset called CAMERA-PRIMUS, which demonstrates that our approach surpass the state-of-the-art end-to-end method using Convolutional Recurrent Neural Network.
\end{abstract}

\section{CCS CONCEPTS}

- Computing methodologies $\rightarrow$ Neural networks.

\section{KEYWORDS}

optical music recognition, music techonology, neural networks, deep learning

\footnotetext{
${ }^{*}$ Corresponding Author.

Permission to make digital or hard copies of all or part of this work for personal or classroom use is granted without fee provided that copies are not made or distributed for profit or commercial advantage and that copies bear this notice and the full citation on the first page. Copyrights for components of this work owned by others than ACM must be honored. Abstracting with credit is permitted. To copy otherwise, or republish, to post on servers or to redistribute to lists, requires prior specific permission and/or a fee. Request permissions from permissions@acm.org.

MMPT '21, August 21, 2021, Taipei, Taiwan

(C) 2021 Association for Computing Machinery.

ACM ISBN 978-1-4503-8530-5/21/08 . .\$15.00

https://doi.org/10.1145/3463945.3469056
}

ACM Reference Format:

Aozhi Liu, Lipei Zhang, Yaqi Mei, Baoqiang Han, Zifeng Cai, Zhaohua Zhu, and Jing Xiao. 2021. Residual Recurrent CRNN for End-to-End Optical Music Recognition on Monophonic Scores. In Proceedings of the 2021 Workshop on Multi-Modal Pre-Training for Multimedia Understanding (MMPT '21), August 21, 2021, Taipei, Taiwan. ACM, New York, NY, USA, 5 pages. https: //doi.org/10.1145/3463945.3469056

\section{INTRODUCTION}

Optical Music Recognition(OMR) is an area of research that investigates how to decode music notations from images. Most of the early studies of Optical Music Recognition have focused on a multi-stage fashion[18]. The complete workflow of the methods normally includes an image prepossessing and image binarization initially[5][21]. Following this step is typically a staff-line detection and removal process. It is worth mentioning that the staff-line detection and removal part is critically important since the failure of this step will result in the failure of the rest parts[6][20]. In the next step, the symbol classification is typically processed to distinguish the musical meaning of each symbol[13][1]. Once these symbols are classified into different categories, they have to be put together to build up the semantic meaning of the music scores[17]. In recent years, as an early attempt of end-to-end system in Optical Music Recognition, Jorge Calvo-Zaragoze et al. implemented a Convolutional Recurrent Neural Network(CRNN) framework proposed by Shi et al.[19] for scene text recognition to solve the OMR problem with deep learning methods[3]. The basic CRNN structure includes several convolutional blocks for extracting features of the images followed by a recurrent block for dealing with the sequential task regarding to the features as well as the output symbols.

However, the feed-forward only convolutional block for the whole CRNN architecture has limitation on the performance of extraction and integration on the contextual information. Inspired by the deep residual model[9] and the RCNN structure applying in 
several computer vision tasks[16][14], we have proposed a Recurrent Residual Convolutional Neural Network for our end-to-end Optical Music Recognition task. The recurrent neural network has been implemented as the subsequent block of the recurrent residual convolutional block for the entire end-to-end system. The recurrent residual convolutional block ensures better feature representation and wider range of contextual information derivation for the OMR system. Moreover, we have evaluated the performance of our proposed model with the dataset called CAMERA-PRIMUS[2] containing both the printed image and as well as the image with distortion. The experiments demonstrate that the results of our end-to-end framework with recurrent residual convolutional blocks outperform the results of the vanilla CRNN framework[3][2], which indicates that our proposed model have achieved the state-of-the-art performance in end-to-end monophonic score recognition system.

\section{APPROACH}

\subsection{Data Representation}

It is well known that the tasks of deep learning require dataset with sufficient size as well as good quality. For these requirements, we implement the Camera-based Printed Images of Music Staves(CameraPrIMuS)[2] dataset for our end-to-end training system. This dataset contains 87678 clean images of music score and the same number of synthetic distorted images to resemble the camera-based scenario.

For each image, there are two kinds of representations to fulfill the end-to-end training purpose of the deep neural network. The examples are shown in Fig. 1. One of the representations is called semantic encoding which includes symbols with musical meaning. For instance, ${ }^{b} \mathrm{~B}$ major key signature (see Fig. 1(c)) is notated with the musical meaning "keySignature". On the other hand, the agnostic encoding (see Fig. 1(d)) contains a list of graphic symbols without musical meaning, e.g. a A Major key signature is represented as a sequence of three sharp symbols.

\subsection{Model Architecture}

In this section, we describe our end-to-end framework containing the feature extraction component integrating the deep residual model[9] and the Recurrent Convolutional Neural Networks (RCNN) model. The complete framework is named as Residual Recurrent Convolutional Recurrent Neural Networks (R2-CRNN).

2.2.1 Residual Recurrent Block. The residual recurrent block is an idea integrating the residual model[9] as well as the recurrent convolutional neural network (RCNN)[14] to improve the accuracy of the monophonic optical music recognition task. The residual recurrent convolutional block is shown in Fig. 2 . The $1 \times 1$ convolutional operation[15] is applied to reduce the number of channels of the input. Followed by this operation is 2 recurrent units using convolution. The recurrent convolutional unites are able to enrich the extracted output features by feature accumulation with the recurrent mechanism. The detailed structure of the recurrent convolutional units is shown in Fig. 3. The channels reduction results of the image input will be taken as the input of the first recurrent convolutional unit. In the first time step, a kernel with the size of $3 \times 3$ is used for convolution, followed by a Batch Normalization[11] process to increase the training efficiency and Rectified Linear Unit[7] for activation. Then, the addition of the input of the recurrent convolutional unit and the sequential operation combined with $3 \times 3$ convolution, Batch Normalization and Rectified Linear Unit activation will be processed twice to derive the output of the recurrent convolutional unit. As the final step of the residual recurrent convolutional block, a residual mechanism is applied so that the output of the block is obtained.

2.2.2 End-to-end Architecture. Some previous work has demonstrated that end-to-end recognition of monophonic music score is plausible[4][1][2]. The end-to-end problem is constructed by an input staff image as well as its corresponding sequence of music symbols.

The graphic scheme of the end-to-end framework is shown in Fig. 4. An image of monophonic staff is taken as the input of the Residual Recurrent Convolutional Recurrent Neural Network (R2CRNN) model without any preprocessing steps such as symbol segmentation or staff-line removal. The R2-CRNN model consists of two blocks: the Residual Recurrent Convolutional Neural Network Block (R2-CNN) and the Recurrent Neural Network Block (RNN). Firstly, the image is processed by the R2-CNN block for feature extraction. Secondly, the features extracted by the R2-CNN block are fed into the RNN block, which finally converts the image into a sequence of music symbols. Moreover, a CTC loss function[8] is applied so that there's no need for framewise labelling of the input data. However, the CTC loss is applied only at the training stage. In the prediction step, the framewise R2-CRNN output can be decoded into a sequence of music symbols.

2.2.3 Implementation details. Firstly, the input image is rescaled at a fixed height of 128 pixels. Then, this input will be processed through a R2-CNN block consisting of four residual recurrent units. Each residual recurrent unit has a $1 \times 1$ convolution operator, two recurrent convolution operators with $3 \times 3$ kernel size and a maxpooling down-sampling operator with $2 \times 2$ window size. The output of the R2-CNN block is then taken as the input of two bi-directional LSTM[10] layers of 256 neurons. Considering both the input features and the modeling of the musical representation, the bi-directioanl LSTM will produce the discrete musical symbol sequence. Finally, a fully-connected layer with a softmax activation function is used for the classification of the music symbol class of each frame. To learn the model, the parameters of the R2-CNN block as well as the Recurrent block are updated repeatedly through the back-propagation using Adam optimizer[12] to minimize the CTC loss function. The batch size to train the model is set to be 16 .

At the stage of prediction, the decoding process can be performed greedily due to the implementation of CTC loss function. If a predicted symbol of a certain frame is the same as the previous one, it is considered the symbol that covers more than one frame. Hence, this symbol will not be appended to the decoding sequence of symbols. On the other hand, if the predicted symbol is different from the previous one or the predicted symbol is the blank symbol, it will be concatenated to the music symbol decoding sequence. 


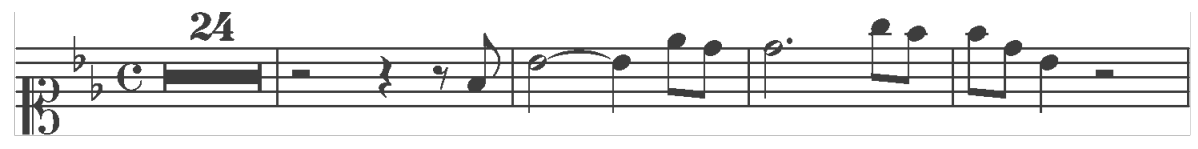

(a) Clean image

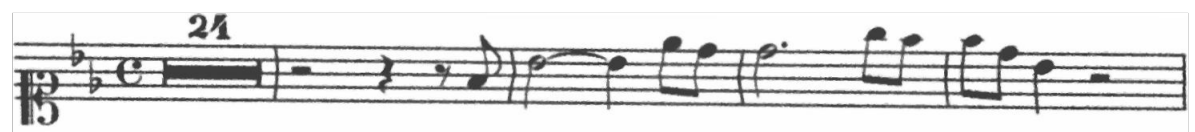

(b) Distorted image

\begin{abstract}
clef-C1, keySignature-BbM, timeSignature-C, multirest-24, barline, rest-half, rest-quarter, rest-eighth, note-F4 eighth, barline, note-Bb4_half, tie, note-Bb4_quarter, note-Eb5_eighth, note-D5_eighth, barline, note-D5_half., note-G5_eighth, note-F5_eighth, barline, note-F5_eighth, note-D5_eighth, note-Bb4_quarter, rest-half, barline
\end{abstract}

(c) Semantic encoding

clef.C-L1, accidental.flat-L4, accidental.flat-L2, metersign.C-L3, digit.2-S5, digit.4-S5, multirest-L3 barline-L1, rest.half-L3, rest.quarter-L3, rest.eighth-L3, note.eighth-S2, barline-L1, note.half-L4, slur.start-L4, slur.end-L4, note.quarter-L4, note.beamedRight1-S5, note. beamedLeft1-L5, barline-L1, note.half-L5, dot-S5, note.beamedRight1-S6, note. beamedLeft1L6, barline-L1, note.beamedRight1-L6, note.beamedLeft1-L5, note.quarter-L4, rest.half-L3, barline-L1

(d) Agnostic encoding

Figure 1: Camera-PrIMus incipit contents example. (a)Example of the clean image. (b)Example of the distorted image (c)Example of semantic encoding (d)Example of agnostic encoding

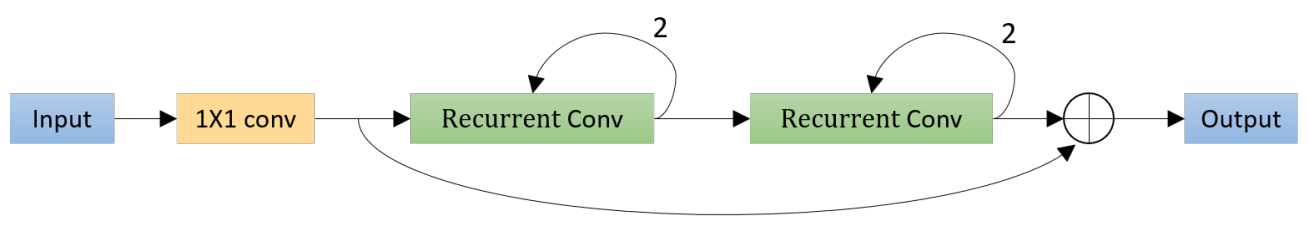

Figure 2: The Structure of Residual Recurrent Convolutional Block.

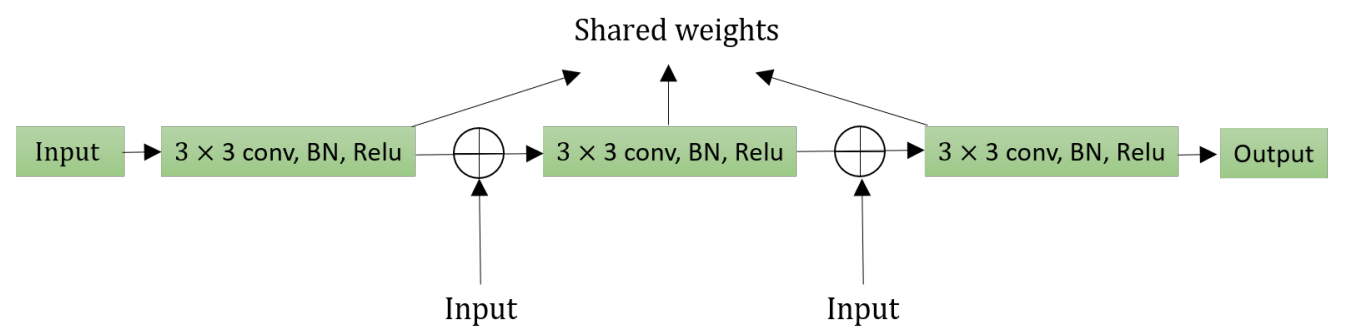

Figure 3: Unfolded Recurrent Convolutional Unit.

\section{EXPERIMENT}

\subsection{Experiment step}

We would like to perform experiments to demonstrate that our model has achieved the state-of-the-art performances in the field of monophonic optical music recognition. For this purpose, the evaluation approach that has been implemented in the previous end-to-end OMR tasks[3][2] is implemented in our work. In this way, we use two quantitative metrics that take into account both the sequence aspect and the symbol aspect:
- Sequence Error Rate (ER) (\%): ratio of incorrectly predicted sequences (at least one error)/

- Symbol Error Rate (SER) (\%): the average number of editing operations such as insertions, deletions, or substitutions that are necessary to generate the reference sequence from the one predicted by the model, normalized by its length.

In Calvo-Zaragoza J.'s paper[2], for both the clean images and the distorted images the ER as well as the SER values are evaluated on the results with both the agnostic labelling and the semantic notation. For comparing our results to this work, which is the most 


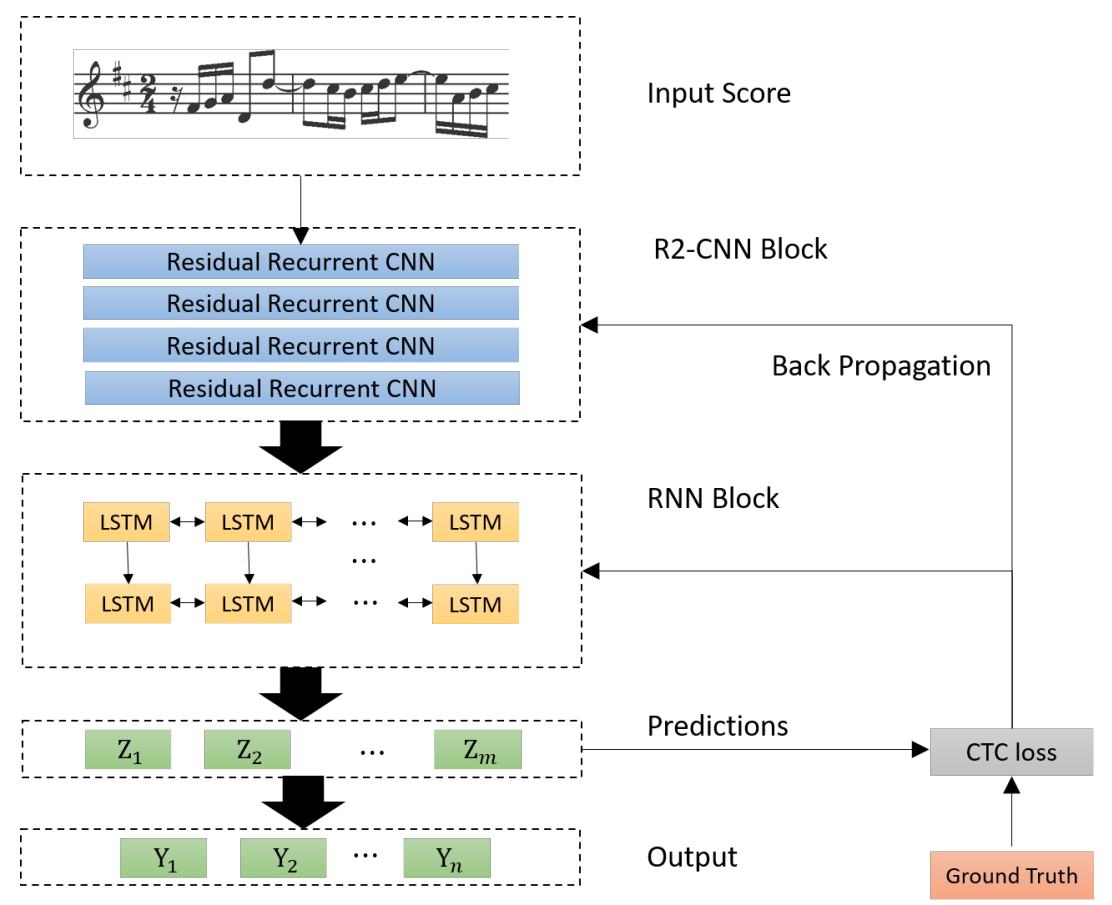

Figure 4: Graphical scheme of the R2-CRNN end-to-end model.

Table 1: Average SER (\%) / ER (\%) values in all possible combinations of training and evaluation conditions.

\begin{tabular}{c|c|c|cccc}
\hline & & & \multicolumn{4}{|c}{ Evaluation } \\
\hline \multirow{3}{*}{} & & & \multicolumn{2}{|c}{ Clean } & \multicolumn{2}{c}{ Distortions } \\
\hline \multirow{3}{*}{ Training } & \multirow{2}{*}{ Clean } & CRNN results & $1.10 / 21.7$ & $0.80 / 12.5$ & $44.3 / 95.1$ & $59.7 / 97.9$ \\
& & Our results & $\mathbf{0 . 5 9 / 1 6 . 2}$ & $\mathbf{0 . 5 6 / 1 3 . 4}$ & $7.63 / \mathbf{2 0 . 9}$ & $\mathbf{3 8 . 1 9 / 9 1 . 0}$ \\
& \multirow{3}{*}{ Distortions } & CRNN results & $1.40 / 24.9$ & $3.3 / 44.6$ & $1.6 / 24.7$ & $3.4 / 38.3$ \\
& & Our results & $\mathbf{0 . 6 8 / 1 8 . 7}$ & $\mathbf{1 . 0 6 / 2 5 . 4}$ & $\mathbf{1 . 0 1 / 2 7 . 8}$ & $\mathbf{1 . 5 0 / 3 5 . 8}$ \\
\hline
\end{tabular}

up-to-date end-to-end framework in OMR previous to our study, we evaluate all metrics in this paper to show the state-of-the-art performance of our model.

\subsection{Performance}

In this section, we show the results of our experiments that have compared with the results of Calvo-Zaragoza J.'s work[2]. We consider three different data partitions of the dataset that $80 \%$ of the data for training while $10 \%$ for validation. The remaining $10 \%$ is used for test.

Calvo-Zaragoza J.'s work[2] reported all possible results regarding the training and evaluation scenarios. To comprehensively evaluate the performances of our model, we also perform the test on all of these conditions. The detailed comparison is shown in the Table. 1. The most notable difference is in the condition that training on clean images while evaluation on distorted images with agnostic notation. The SER (\%) value and the ER (\%) value are 44.3 and 95.1 respectively when using CRNN model. With our model, the SER
(\%) value and the ER (\%) value are only 7.63 and 20.9. Actually, in all conditions the results of our model surpass the performances of the Convolutional Recurrent Neural Network (CRNN) model implemented by Calvo-Zaragoza J. which reflect that our R2-CRNN model has significant improvement of the up-to-date CRNN model on the monophonic Optical Music Recognition tasks.

\section{CONCLUSION}

In this work, we have proposed an innovative end-to-end framework for Optical Music Recognition consisting a Residual Recurrent Convolution block as well as a Recurrent Neural Network block. Compared to early end-to-end model, our improvement enhanced the capability of the kernel filters to capture statistical features in the context of the symbols.The results of experiments demonstrate that our model outperform the up-to-date model(CRNN) architecture, which have achieved the state-of-the-art results of the tasks of monophonic Optical Music Recognition. 


\section{REFERENCES}

[1] Jorge Calvo-Zaragoza, Antonio-Javier Gallego, and Antonio Pertusa. 2017. Recognition of handwritten music symbols with convolutional neural codes. In 2017 14th IAPR International Conference on Document Analysis and Recognition (ICDAR), Vol. 1. IEEE, 691-696.

[2] Jorge Calvo-Zaragoza and David Rizo. 2018. Camera-PrIMuS: Neural End-to-End Optical Music Recognition on Realistic Monophonic Scores.. In ISMIR. 248-255.

[3] Jorge Calvo-Zaragoza and David Rizo. 2018. End-to-end neural optical music recognition of monophonic scores. Applied Sciences 8, 4 (2018), 606

[4] Jorge Calvo-Zaragoza, Jose J Valero-Mas, and Antonio Pertusa. 2017. End-toend optical music recognition using neural networks. In Proceedings of the 18th International Society for Music Information Retrieval Conference, ISMIR. 23-27.

[5] Jorge Calvo-Zaragoza, Gabriel Vigliensoni, and Ichiro Fujinaga. 2017. Pixel-wise binarization of musical documents with convolutional neural networks. In 2017 Fifteenth IAPR International Conference on Machine Vision Applications (MVA) IEEE, 362-365.

[6] Alicia Fornes, Anjan Dutta, Albert Gordo, and Josep Llados. 2011. The ICDAR 2011 music scores competition: Staff removal and writer identification. In 2011 International Conference on Document Analysis and Recognition. IEEE, 1511-1515.

[7] Xavier Glorot, Antoine Bordes, and Yoshua Bengio. 2011. Deep sparse rectifier neural networks. In Proceedings of the fourteenth international conference on artificial intelligence and statistics. 315-323.

[8] Alex Graves, Santiago Fernández, Faustino Gomez, and Jürgen Schmidhuber 2006. Connectionist temporal classification: labelling unsegmented sequence data with recurrent neural networks. In Proceedings of the $23 \mathrm{rd}$ international conference on Machine learning. 369-376.

[9] Kaiming He, Xiangyu Zhang, Shaoqing Ren, and Jian Sun. 2016. Deep residual learning for image recognition. In Proceedings of the IEEE conference on computer vision and pattern recognition. $770-778$.

[10] Sepp Hochreiter and Jürgen Schmidhuber. 1997. Long short-term memory. Neural computation 9, 8 (1997), 1735-1780.
[11] Sergey Ioffe and Christian Szegedy. 2015. Batch normalization: Accelerating deep network training by reducing internal covariate shift. arXiv preprint arXiv:1502.03167 (2015)

[12] Diederik P Kingma and Jimmy Ba. 2014. Adam: A method for stochastic optimization. arXiv preprint arXiv:1412.6980 (2014)

[13] Sangkuk Lee, Sung Joon Son, Jiyong Oh, and Nojun Kwak. 2016. Handwritten music symbol classification using deep convolutional neural networks. In 2016 International Conference on Information Science and Security (ICISS). IEEE, 1-5.

[14] Ming Liang and Xiaolin Hu. 2015. Recurrent convolutional neural network for object recognition. In Proceedings of the IEEE conference on computer vision and pattern recognition. 3367-3375.

[15] Min Lin, Qiang Chen, and Shuicheng Yan. 2013. Network in network. arXiv preprint arXiv:1312.4400 (2013).

[16] Pedro Pinheiro and Ronan Collobert. 2014. Recurrent convolutional neural networks for scene labeling. In International conference on machine learning. 82-90.

[17] Christopher Raphael and Jingya Wang. 2011. New Approaches to Optical Music Recognition.. In ISMIR. 305-310.

[18] Ana Rebelo, Ichiro Fujinaga, Filipe Paszkiewicz, Andre RS Marcal, Carlos Guedes, and Jaime S Cardoso. 2012. Optical music recognition: state-of-the-art and open issues. International Journal of Multimedia Information Retrieval 1, 3 (2012), 173-190.

[19] Baoguang Shi, Xiang Bai, and Cong Yao. 2016. An end-to-end trainable neural network for image-based sequence recognition and its application to scene Text recognition. IEEE transactions on pattern analysis and machine intelligence 39,11 (2016), 2298-2304.

[20] Bolan Su, Shijian Lu, Umapada Pal, and Chew Lim Tan. 2012. An effective staff detection and removal technique for musical documents. In 2012 10th IAPR International Workshop on Document Analysis Systems. IEEE, 160-164.

[21] Quang Nhat Vo, Soo Hyung Kim, Hyung Jeong Yang, and Gueesang Lee. 2016. An MRF model for binarization of music scores with complex background. Pattern Recognition Letters 69 (2016), 88-95. 